Supporting Information for:

\title{
The Underlying Chemical Mechanism of Selective Chemical Etching in CsPbBr3 Nanocrystals for Reliably Accessing Near-Unity Emitters
}

\author{
Brent A. Koscher ${ }^{1,3,4}$, Zachary Nett1,3,4, and A. Paul Alivisatos, $1,2,3,4$ \\ ${ }^{1}$ Department of Chemistry, University of California, Berkeley, CA 94720, USA. \\ 2 Department of Materials Science and Engineering, University of California, Berkeley, CA 94720, USA. \\ 3 Materials Science Division, Lawrence Berkeley National Laboratory, Berkeley, CA 94720, USA. \\ 4 Kavli Energy NanoScience Institute, Berkeley, CA 94720, USA.
}

\section{Materials:}

Cesium carbonate $\left(\mathrm{Cs}_{2} \mathrm{CO}_{3}, 99.9 \%\right.$, Aldrich), octadecene (ODE, 90\%, Aldrich), oleic acid (OA, 90\%, Aldrich), oleylamine (OLA, 70\%, Aldrich), lead bromide ( $\mathrm{PbBr}_{2}, 99.999 \%$, Aldrich), hexanes (>99\%, Aldrich, anhydrous), toluene (99.8\%, Aldrich, anhydrous), ethyl acetate (99.8\%, Aldrich, anhydrous), ammonium thiocyanate $\left(\mathrm{NH}_{4} \mathrm{SCN}, 99.99 \%\right.$, Aldrich), sodium thiocyanate (NaSCN, 99.99\%, Aldrich), lead thiocyanate (99.5\%, Aldrich), octanedithiol (>97\%, Aldrich), sodium diethyldithiocarbamate ( $>99 \%$, Aldrich), potassium thioacetate (98\%, Aldrich), potassium selenocyanate (>99\%, Aldrich), tetramethylthiourea (98\%, Aldrich)

\section{Synthetic Methods:}

\section{Preparation of Cesium Oleate Stock Solution}

A stock solution of cesium oleate was prepared following the reported procedure by Protesescu et al. (Ref 16). Briefly, $2.5 \mathrm{mmol} \mathrm{Cs}_{2} \mathrm{CO}_{3}$ and $2.5 \mathrm{~mL} \mathrm{OA}$ was loaded into a three-neck flask containing $40 \mathrm{~mL}$ ODE. The mixture was dried/degassed for 1 hour at $120^{\circ} \mathrm{C}$ under high vacuum, then heated under dry Argon gas at $150^{\circ} \mathrm{C}$ until all the $\mathrm{Cs}_{2} \mathrm{CO}_{3}$ complexed with OA.

\section{Preparation of Ligands for Synthesis}

Small quantities of OA and OLA (typically on the 5-10 mL scale) were prepared ahead of time for use in the synthesis. The technical grade OA and OLA liquids were filtered through a PTFE syringe filter to remove any large floating particulates and then loaded into separate glass vials. Then OA and OLA were dried/degassed for 1 hour at $120{ }^{\circ} \mathrm{C}$ and then transferred air-free into an argon glovebox for storage. Afterwards the OA and OLA were used without further processing.

\section{Synthesis of CsPbBr ${ }_{3}$ Nanocrystal Cubes}

First ODE $(5 \mathrm{~mL})$ and $\mathrm{PbBr}_{2}(0.188 \mathrm{mmol})$ were added into a three-neck flask, then dried/degassed under vacuum at $120^{\circ} \mathrm{C}$ for 1 hour. Afterwards the reaction was heated under dry argon gas to $140{ }^{\circ} \mathrm{C}$, at which point previously prepared OA $(0.5 \mathrm{~mL})$ and OLA $(0.65 \mathrm{~mL})$ were injected into the flask to complex the $\mathrm{PbBr}_{2}$ salt. Following the complete dissolution of the $\mathrm{PbBr}_{2}$ salt, the reaction temperature was adjusted between $140-160{ }^{\circ} \mathrm{C}$ before hot cesium oleate solution ( $0.5 \mathrm{~mL}, 0.125 \mathrm{M}$ ) was injected. The size of the obtained particles is modulated by changing the reaction temperature. After growing for 5-10 seconds, the reaction mixture was cooled close to room-temperature using an ice-water bath.

\section{Isolation and Purification of $\mathrm{CsPbBr}_{3}$ Nanocrystal Cubes}

Three isolation methods work to good effect for 7-10 nm edge length $\mathrm{CsPbBr}_{3}$ nanocrystal cube samples. In the first method, the crude reaction mixture was first centrifuged at $2000 \mathrm{RCF}$ for 3 minutes to remove aggregates and other undesirably large by-products, then centrifuged at $11000 \mathrm{RCF}$ for up to 10 minutes at low temperatures $\left(0-10{ }^{\circ} \mathrm{C}\right)$, before finally re-dispersing the isolated particles in anhydrous hexanes for storage. In the second method, the crude solution was first centrifuged at $2000 \mathrm{RCF}$ for 3 minutes to remove unwanted by-products, before adding ethyl acetate (or similar anti-solvent), centrifuging the resulting 
suspension at $9000 \mathrm{RCF}$ for up to 10 minutes, and finally re-dispersing the isolated particles in anhydrous hexanes for storage. In the third method, around $5 \mathrm{~mL}$ of hexanes were added to the crude reaction mixture and centrifuged at 2000 RCF to remove unwanted by-products. Next, hexanes were evaporated from the mixture in a step-wise fashion in order to isolate different size particles from the reaction solution by centrifugation at $9000 \mathrm{RCF}$ for up to 10 minutes, and then the isolated particles were re-dispersed in anhydrous hexanes for storage. Each method results in long-term stable particles that can be stored in an inert atmosphere glovebox or in air, depending on the timeframe in which the samples were used.

\section{Synthesis of CsPbBr ${ }_{3}$ Nanocrystal Plates}

First ODE $(5 \mathrm{~mL})$ and $\mathrm{PbBr}_{2}(0.188 \mathrm{mmol})$ were added into a three-neck flask, then dried/degassed under vacuum at $120^{\circ} \mathrm{C}$ for 1 hour. Afterwards the reaction was heated under dry argon gas to $140{ }^{\circ} \mathrm{C}$, at which point previously prepared OA $(0.5 \mathrm{~mL})$ and OLA $(0.5 \mathrm{~mL})$ were injected into the flask to complex the $\mathrm{PbBr}_{2}$ salt. Following the complete dissolution of the $\mathrm{PbBr}_{2}$ salt, the reaction temperature was adjusted between 90-130 ${ }^{\circ} \mathrm{C}$ before hot cesium oleate solution (0.4 mL, $0.125 \mathrm{M}$ ) was injected. The size of the obtained particles is modulated by changing the reaction temperature. After growing for 5-10 seconds, the reaction mixture was cooled close to room-temperature using an ice-water bath.

\section{Isolation and Purification of $\mathrm{CsPbBr}_{3}$ Nanocrystal Plates}

To isolate the nanocrystal plates, the reaction mixture was centrifuged at $9000 \mathrm{RCF}$ for up to 10 minutes to yield a precipitate that was re-dispersed in hexanes. Decreasing the temperature during centrifugation can further help with isolation but is often not necessary for nanocrystal plates due to their larger volume. The nanocrystal plate samples were stored inside the glovebox or in air, depending on the timeframe in which the samples were used.

\section{Determination of Fraction of $\mathrm{CsPbBr}_{3}$ Remaining Throughout Etching}

To determine the amount of $\mathrm{CsPbBr}_{3}$ remaining as the etching reaction progressed, the time-dependent steady-state absorption spectra were analyzed. There are two species to monitor, $\mathrm{CsPbBr}_{3}$ and the leaddepleted phase $\mathrm{Cs}_{4} \mathrm{PbBr}_{6}$ material. For the $\mathrm{Cs}_{\mathrm{PbBr}}$ material there are two absorption regions that have no size-dependent features: $325-345 \mathrm{~nm}$ and $415-455 \mathrm{~nm}$. To avoid contributions from $\mathrm{Cs}_{4} \mathrm{PbBr}_{6}$ we monitor the change in absorption near $425 \mathrm{~nm}$ and correlate the amount of $\mathrm{CsPbr}_{3}$ present using the absorption coefficient.

\section{Additional Supporting Figures:}

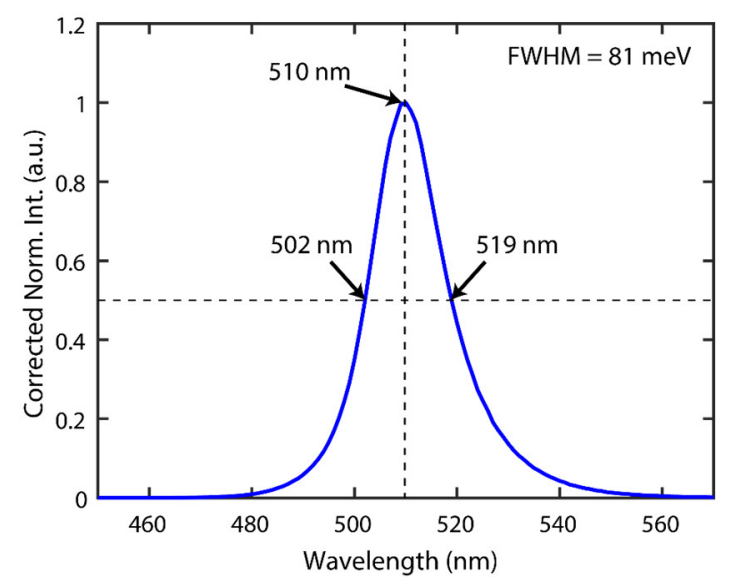

Figure S1: Photoluminescence spectra of the typical $\mathrm{CsPbBr}_{3}$ nanocrystal cubes as-isolated from the reaction mixture. The linewidth of the ensemble is $17 \mathrm{~nm}(81 \mathrm{meV})$ and centered at $510 \mathrm{~nm}$. 


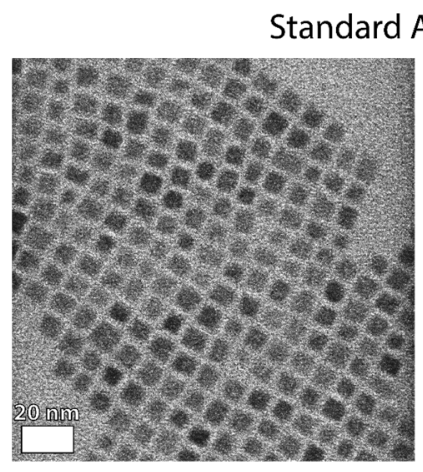

Pre-Treatment

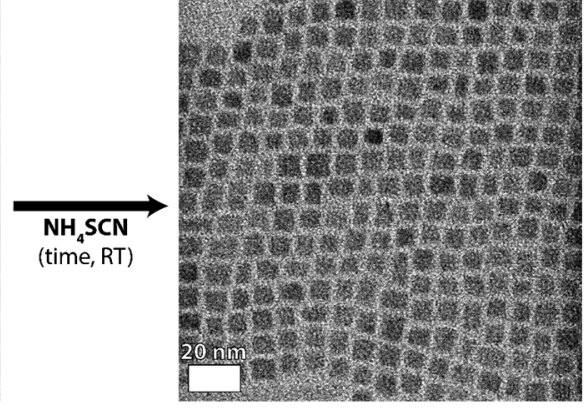

Post-Treatment

Figure S2: For as-isolated $\mathrm{CsPbBr}_{3}$ nanocrystal cubes, with the as-isolated ligand concentration, there is very little, if any, change in the particle size or shape following a room temperature treatment with ammonium thiocyanate, highlighted in the TEM images of the samples.

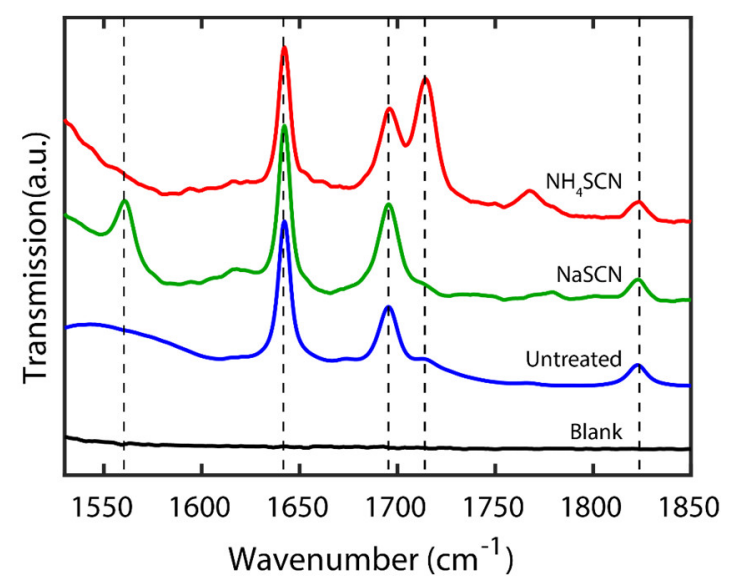

Figure S3: Infrared spectra of the liquid samples in the $1530 \mathrm{~cm}^{-1}$ to $1850 \mathrm{~cm}^{-1}$ wavenumber region. Representative samples included in the plot are the hexanes blank, untreated $\mathrm{CsPBB}_{3}$ nanocrystal cubes, sodium thiocyanate treated sample, and ammonium thiocyanate treated sample. When treating with sodium thiocyanate there is a characteristic peak that appears at $1560 \mathrm{~cm}^{-1}$, and when treating with ammonium thiocyanate there is a characteristic peak that appears at $1712 \mathrm{~cm}^{-1}$. 


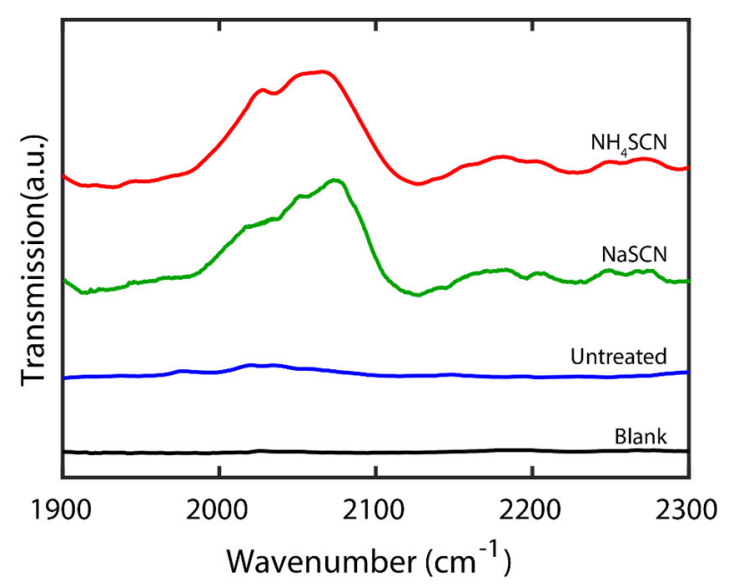

Figure S4: Infrared spectra of the liquid samples in the $1900 \mathrm{~cm}^{-1}$ to $2300 \mathrm{~cm}^{-1}$ wavenumber region. Representative samples included in the plot are the hexanes blank, untreated $\mathrm{CsPbBr}_{3}$ nanocrystal cubes, sodium thiocyanate treated sample, and ammonium thiocyanate treated sample. The treatment results in characteristic vibrational peaks that appear around $2060 \mathrm{~cm}^{-1}$ that correspond to the cyano- stretching mode when bound to lead.
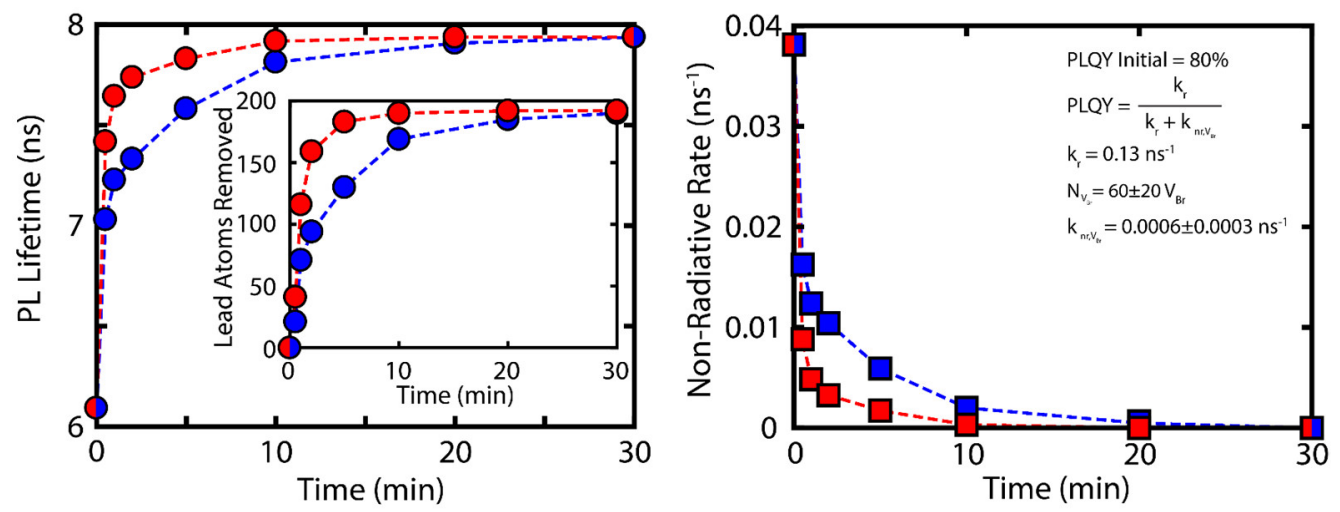

Figure S5: Determination of the non-radiative contribution per bromine vacancy, resulting in an undercoordinated lead atom that acts as a shallow electron state for a $\mathrm{CsPbBr}$ sample with an initial PLQY of 80 $\pm 2.5 \%$. The combination of photoluminescence excited state lifetime, the number of lead atoms removed from the average nanocrystal determined using quantitative IR, and photoluminescence quantum yield allows us to monitor the progress of the etching. This was conducted using both ammonium (red lines) and sodium thiocyanate (blue lines). We assume that the defect sites act independently of each other, i.e. they linearly contribute to the non-radiative rate $\left(k_{n r, T o t a l}=N_{V_{B r}} k_{n r, V_{B r}}\right)$. In order to capture the change in PL Lifetime with a linear contribution of each defect state, we find that more lead atoms are removed than there are under-coordinated lead atoms present. By combining these measurements, we determined the number of bromine vacancies $\left(N_{V_{B r}}\right)$ and non-radiative contribution per bromine vacancy $\left(k_{n r, V_{B r}}\right)$. While there are $\sim 190$ lead atoms removed during the etching reaction under as-isolated ligand conditions, this only represents $\sim 3 \%$ of the total number of lead atoms present in the average nanocrystal, strongly suggesting that not all the ligands present in the as-isolated samples are available to participate in the etching reaction. 


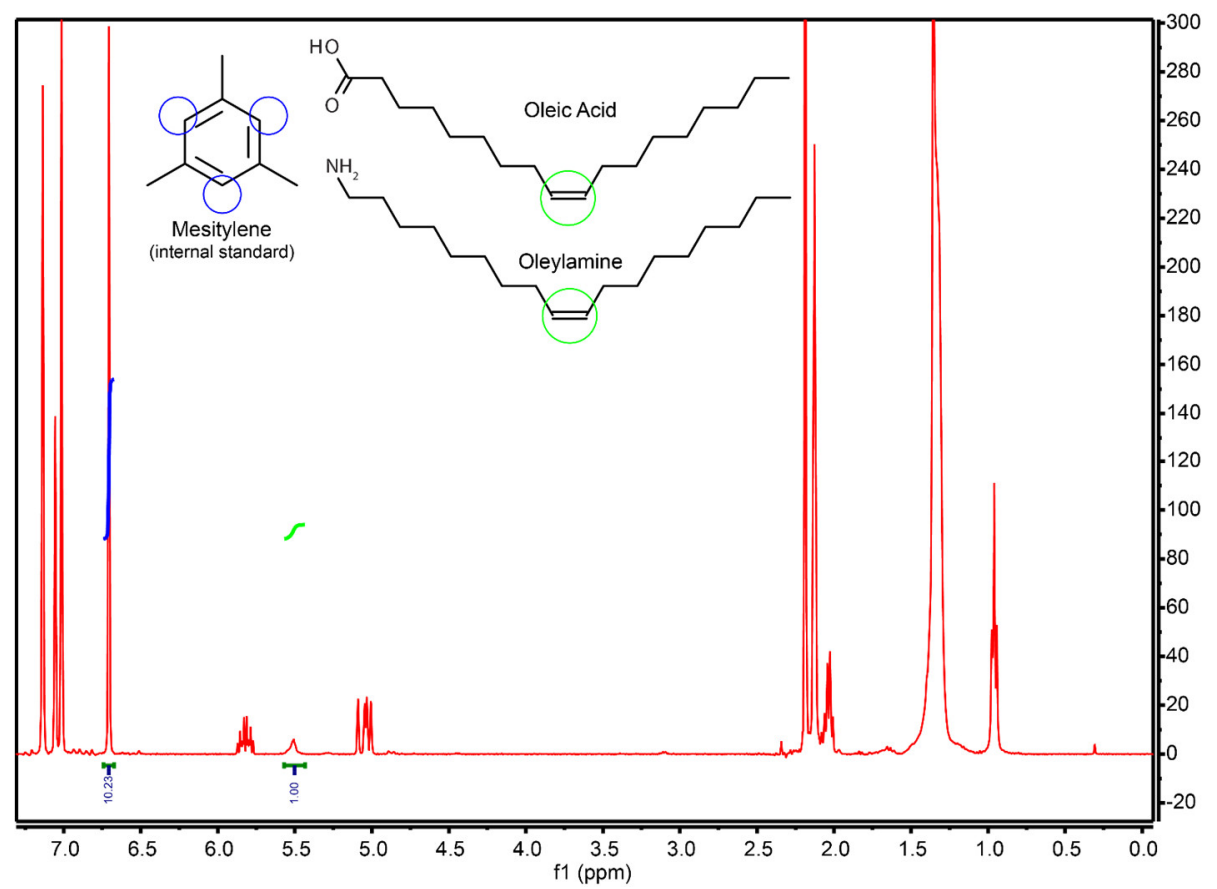

Figure S6: NMR spectra of the typical as-isolated $\mathrm{CsPbBr}_{3}$ nanocrystal cubes. In order to determine the concentration of ligands $(\sim 5.5 \mathrm{ppm})$ present we compare to the mesitylene internal standard $(\sim 6.7 \mathrm{ppm})$. The alkene region contains contributions from both oleic acid and oleylamine. Here we assume that there are equal concentrations of oleic acid and oleylamine present in the solution. For this measurement $5 \mu \mathrm{L}$ of mesitylene was added to a $0.5 \mathrm{~mL}$ solution of 0.75 nanomoles of $\mathrm{CsPbBr}_{3}$ nanocrystal cubes in $\mathrm{d}_{6}$-toluene. For the measurement of typical as-isolated $\mathrm{CsPbBr}_{3}$ nanocrystal cubes, we find that there is around 6 Oleic Acid and 6 Oleylamine ligands present per square nanometer of nanocrystal surface area. However, at these conditions we find that only $\sim 190$ lead atoms from a $10 \mathrm{~nm}$ edge length nanocrystal are removed on average. The removal of 190 lead atoms only represents $\sim 5 \%$ of the number of ligands available in solution. 

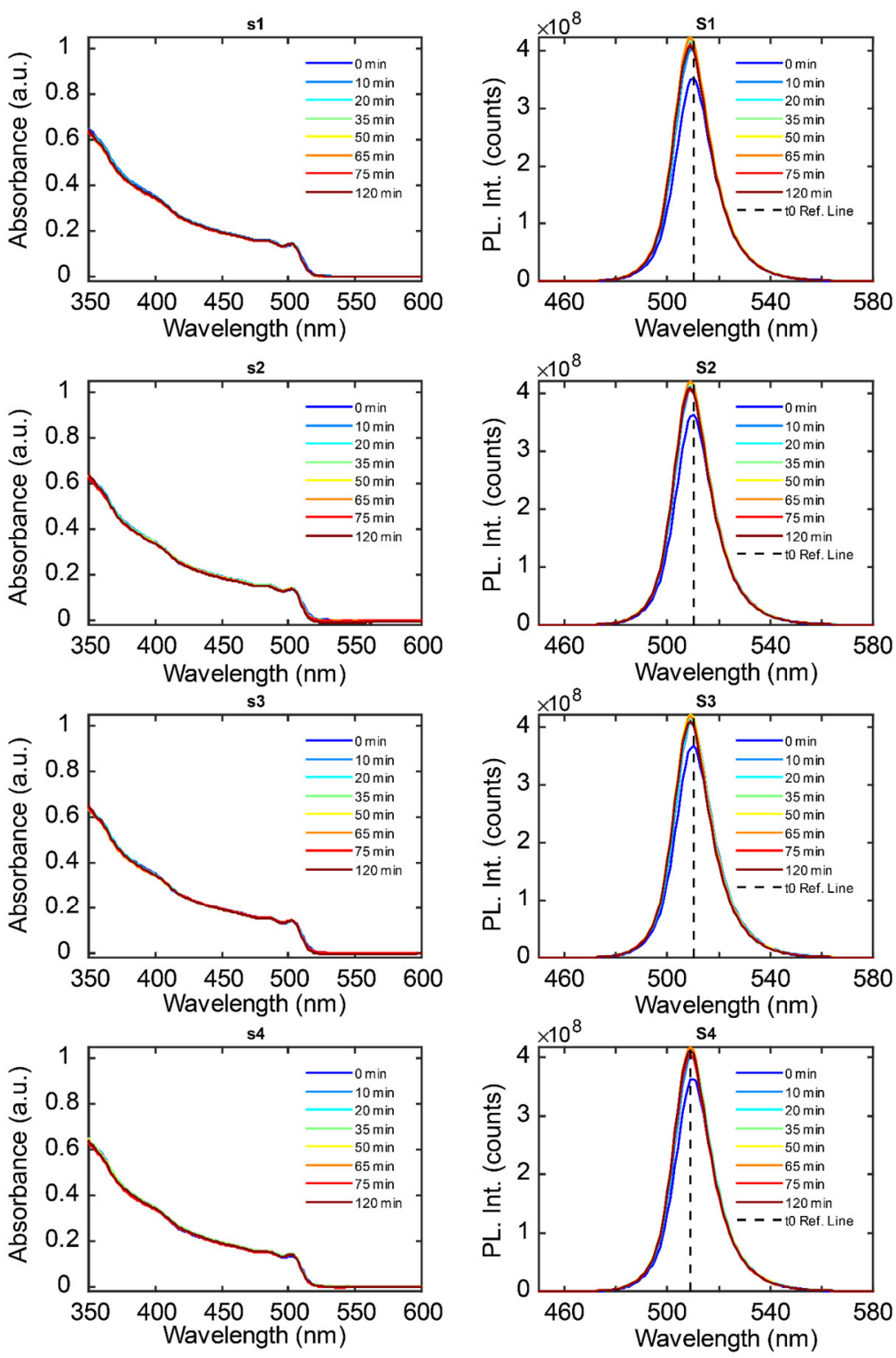

Figure S7: The absorption (left column) and corresponding photoluminescence (right column) spectra from a time series in which each set of spectra shows the as-isolated sample with increasing oleic acid concentration present in solution. Each sample had $\sim 6$ oleylamine $/ \mathrm{nm}^{2}$; then (A) had 8 oleic acid $/ \mathrm{nm}^{2}$, (B) had 10 oleic acid/nm², (C) had 12 oleic acid/nm², and (D) had 14 oleic acid/nm². 

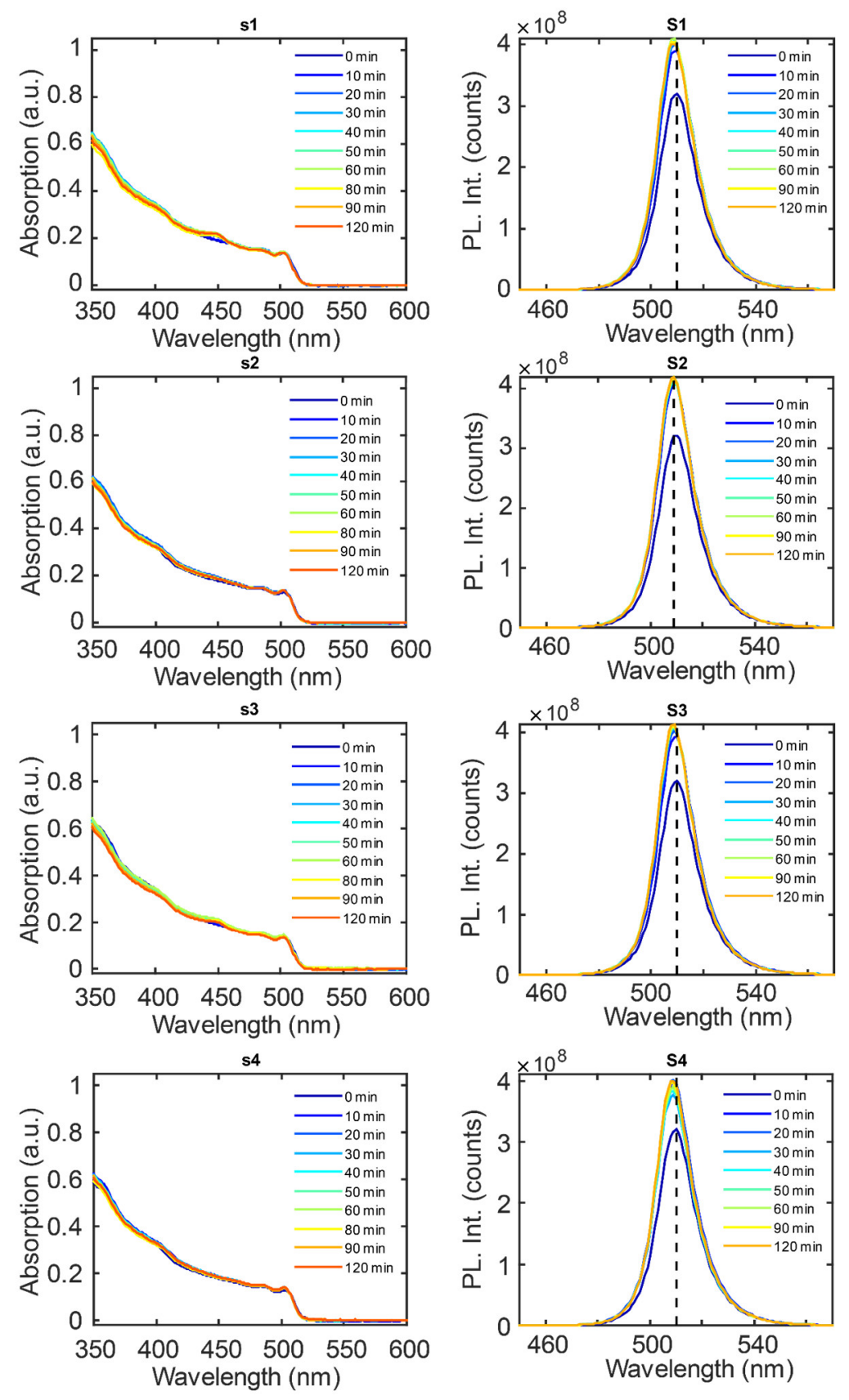

Figure S8: The absorption (left column) and corresponding photoluminescence (right column) spectra from a time series in which each set of spectra shows the as-isolated sample with increasing oleylamine concentration present in solution. Each sample had $\sim 6$ oleic acid $/ \mathrm{nm}^{2}$; then (A) had 8 oleylamine $/ \mathrm{nm}^{2}$, (B) had 10 oleylamine $/ \mathrm{nm}^{2}$, (C) had 12 oleylamine $/ \mathrm{nm}^{2}$, and (D) had 14 oleylamine $/ \mathrm{nm}^{2}$. 

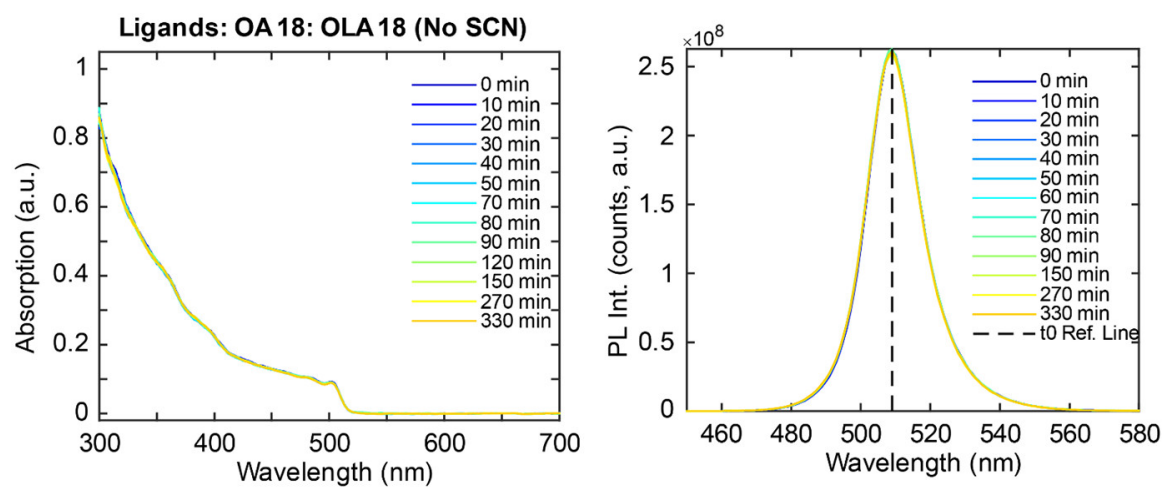

Figure S9: Control run to test the sensitivity of the $\mathrm{CsPBr}_{3}$ nanocrystals to the increased ligand concentration present in solution. Shown are the absorption (left) and the photoluminescence (right) spectra for a time series in which there were 18 oleic acid $/ \mathrm{nm}^{2}$ and 18 oleylamine $/ \mathrm{nm}^{2}$. For these samples no thiocyanate etchant was added into the sample.

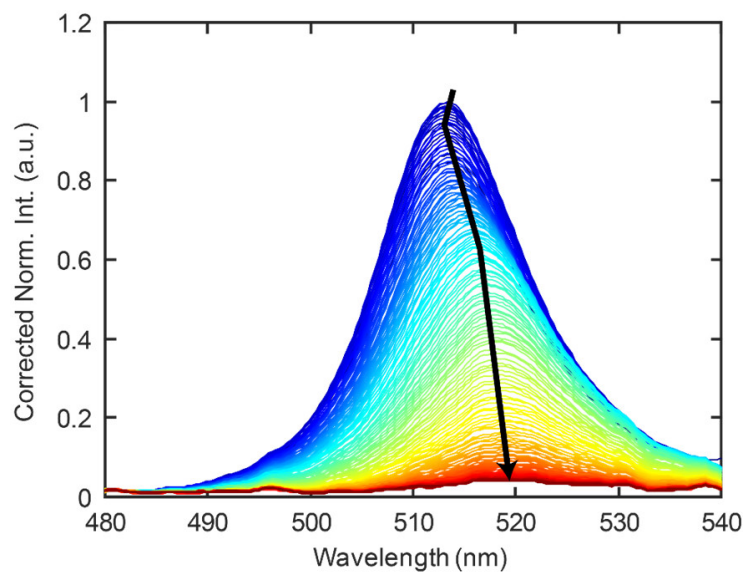

Figure S10: Depiction of the evolution of the photoluminescence spectra of a $\mathrm{CsPbBr}_{3}$ nanocrystal cube sample when etched with thiocyanate. This behavior is typical of a sample that does not have enough ligands present in solution to maintain a high level of colloidal stability as the etching reaction progresses. For this sample there was initially a characteristic blue shift, however the particles began to aggregate and fall out of solution. The characteristic red-shift in the nanoparticle ensemble is indicative of this aggregation. The sample was stirred continuously to ensure no issues arose pertaining to nanoparticles settling to the bottom of the cuvette. 


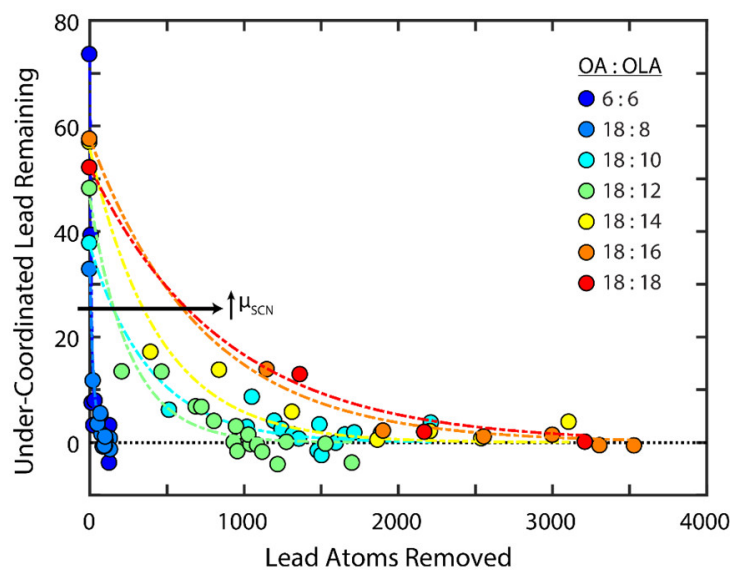

Figure S11: Depiction of the number of under-coordinated lead atoms remaining versus the total number of lead atoms removed under an increasing thiocyanate chemical potential (blue to red). By increasing the chemical potential, the selectivity in the reaction is lost, requiring more lead atoms to be removed from the particles before they remove all the under-coordinated lead atoms. This highlights the need to get the right chemical potential to increase the reaction selectivity.

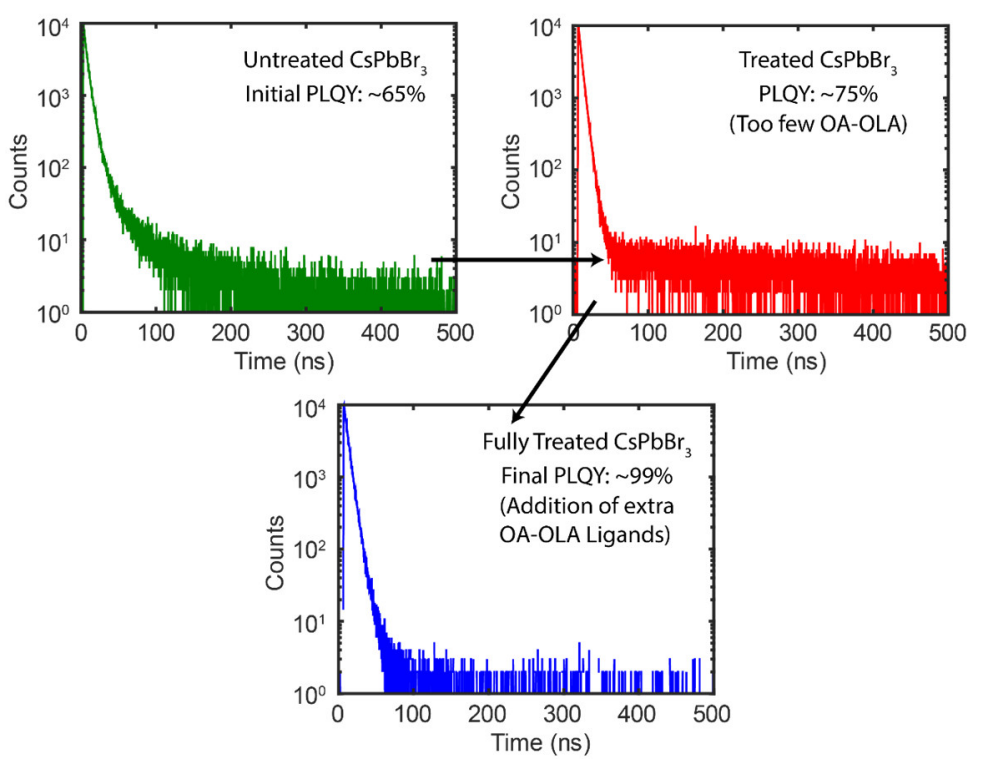

Figure S12: Depiction of the change in the photoluminescence excited state decay and quantum yield for a treatment of $\sim 65 \%$ PLQY particles that have an insufficient number of OA-OLA ion pairs to solubilize enough thiocyanate (green to red), reaching a PLQY of $\sim 75 \%$. The long tail that appears as a second component in the excited state decay is also indicative of incomplete etching. By further increasing the OAOLA ligand concentration in solution we can etch further and reach a final PLQY of near-unity (red to blue). By tailoring the concentration of OA-OLA ion pairs present in solution for the etching, we can take untreated particles and push them to fully treated particles (green to blue). 

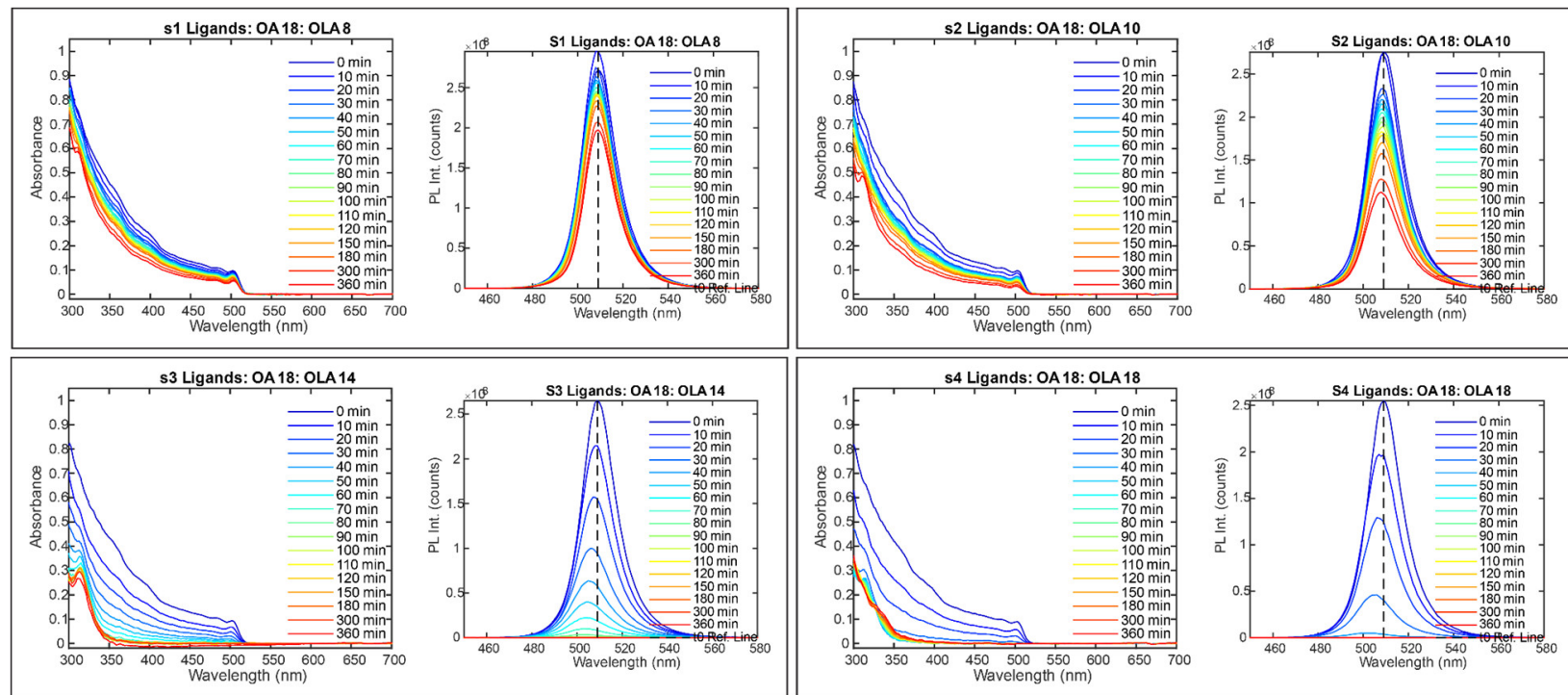

Figure S13: Change in the absorption and photoluminescence spectra for samples etched with thiocyanate containing a constant 18 oleic acid $/ \mathrm{nm}^{2}$ and a varying (A) 8 oleylamine $/ \mathrm{nm}^{2}$, (B) 10 oleylamine $/ \mathrm{nm}^{2}$, (C) 14 oleylamine $/ \mathrm{nm}^{2}$, and (D) 18 oleylamine $/ \mathrm{nm}^{2}$. By increasing the aggressiveness of the etching reaction, we observe a systematically larger blue shift in the photoluminescence bandgap as the etching reaction progresses, indicative of the particle size shrinking throughout the etching reaction.
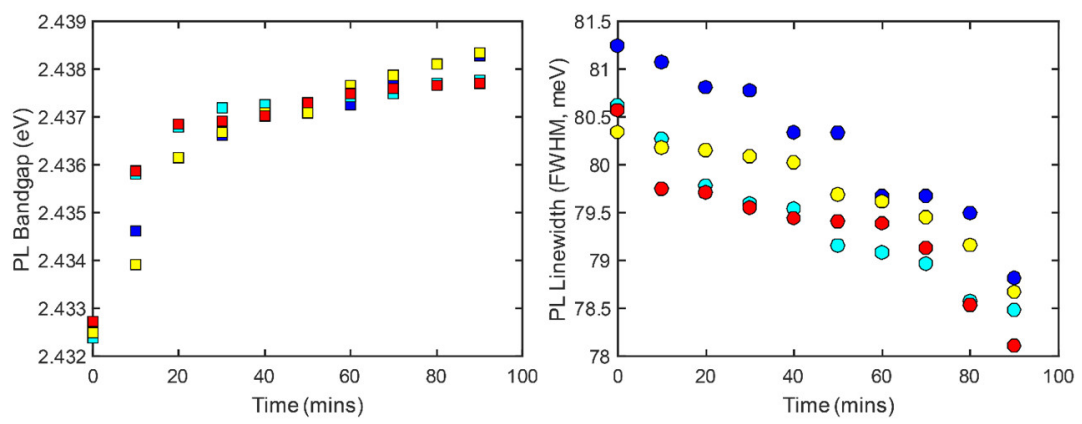

Figure S14: Depiction of the change in the photoluminescence bandgap and the photoluminescence linewidth of four samples of $\mathrm{CsPbBr}_{3}$ exposed to different thiocyanate chemical potentials. In each etching reaction there is a systematic blueshift of the photoluminescence bandgap and subtle narrowing of the photoluminescence linewidth, indicative of not only a subtle etching, but also a size-dependent etching reaction in which larger particles are etched at a faster rate than smaller particles. 


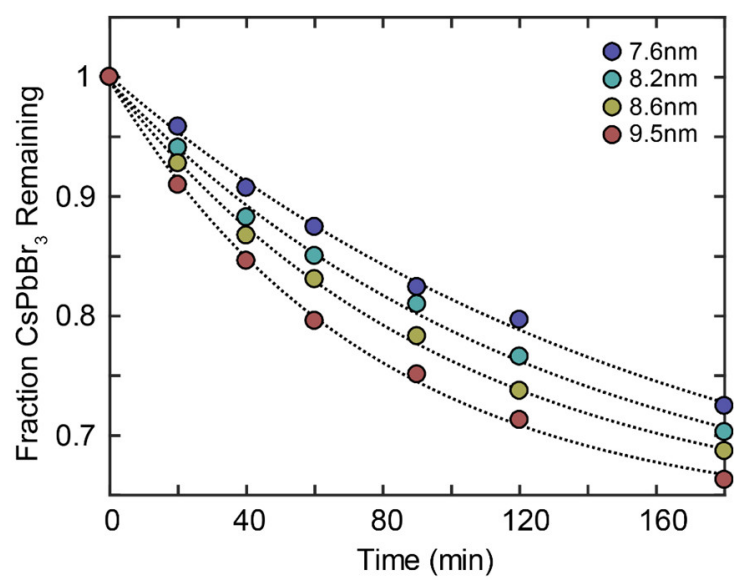

Figure S15: Size-dependent etching rate of $\mathrm{CsPbBr}_{3}$ nanocrystal cubes. Shown are the trajectories of a 7.6 $\mathrm{nm}, 8.2 \mathrm{~nm}, 8.6 \mathrm{~nm}$, and $9.5 \mathrm{~nm}$ average size ensemble exposed to 18 oleic acid/nm ${ }^{2}$ and 12 oleylamine $/ \mathrm{nm}^{2}$.
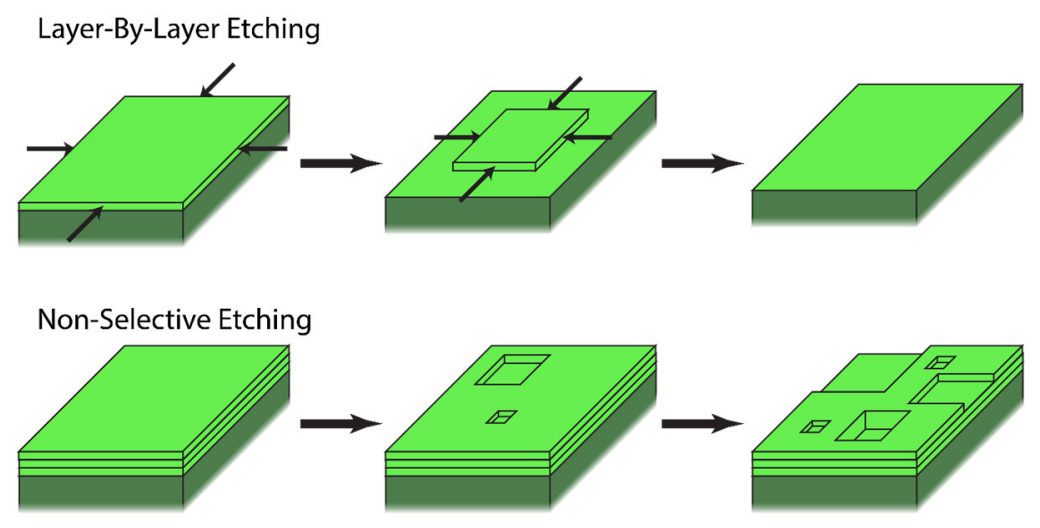

Figure S16: Depiction of the layer-by-layer etching reaction versus a non-selective random etching reaction.
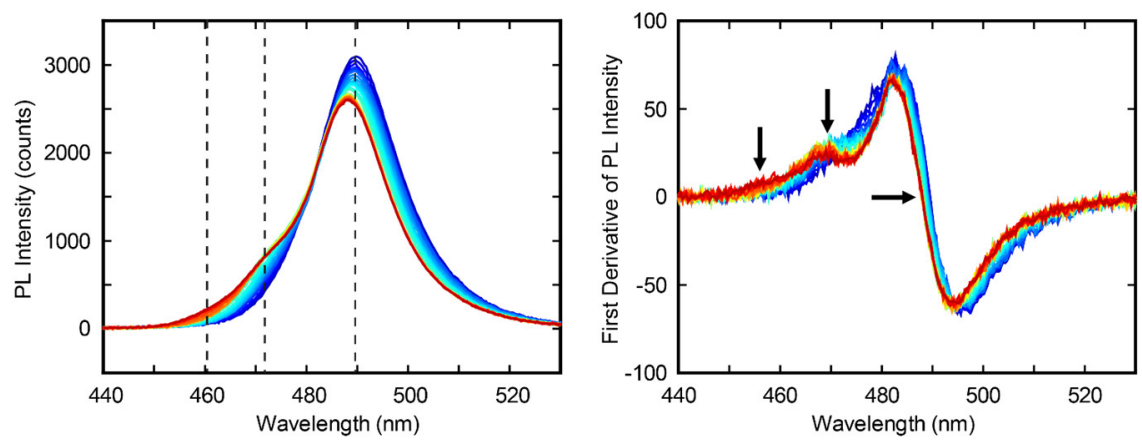

Figure S17: Change in the photoluminescence spectra of a 5-monolayer thick $\mathrm{CsPbBr}_{3}$ nanoplate sample during the etching reaction showing first, the gradual increase in a peak related to the 4-monolayer thick nanoplate, and then later, the same for the 3-monolayer thick nanoplate. The emergence of these peaks can be seen in the first derivative of the photoluminescence spectra in which two clear features and a third subtle feature emerge. 

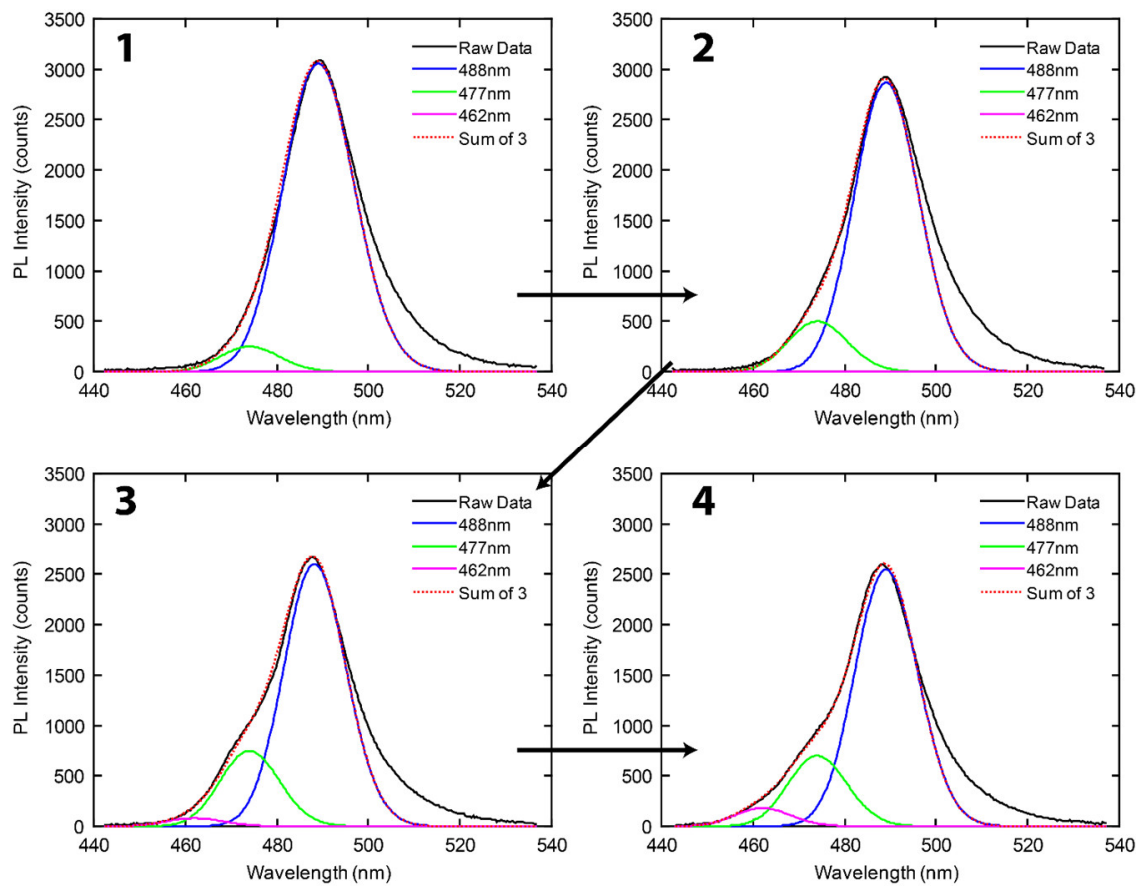

Figure S18: Identified components of the photoluminescence spectra of the ensemble of nanoplates etching in Figure S17. In the spectra there is a related peak centered at $488 \mathrm{~nm}$ (blue line), $477 \mathrm{~nm}$ (green line), and $462 \mathrm{~nm}$ (pink line). The intensities of these peaks are varied to reconstruct the blue-edge of the photoluminescence spectra (dotted red line).

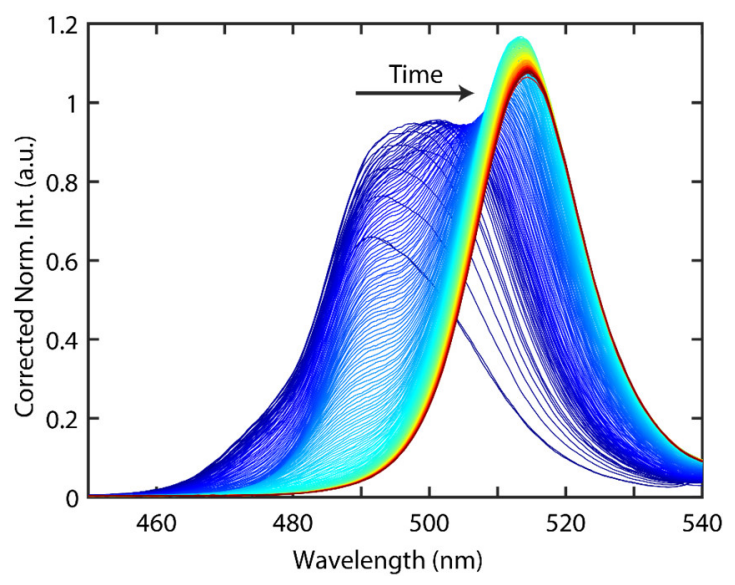

Figure S19: Change in the photoluminescence spectra of a $\mathrm{CsPbBr}_{3}$ nanoplate sample when exposed to too large of an etching chemical potential. The plates quickly aggregate resulting in a substantial redshift of the photoluminescence spectra before the ensemble begins to lose photoluminescence. This highlights the need to carefully etch nanoplates to maintain isolated, well-defined nanoplates. 


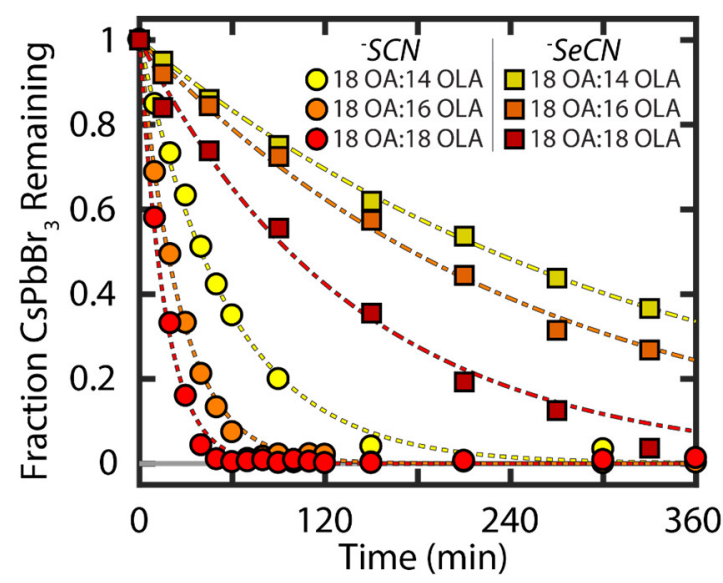

Figure S20: Change in the etching rate of thiocyanate (-SCN) versus selenocyanate (-SeCN) while exposed to the same OA-OLA ion pair concentrations. The selenocyanate is systematically slower than the thiocyanate at the same ligand concentration, consistent with the notion that the chemical potential of the etchant is crucial in dictating the extent of etching and is modulated by the hardness or softness of the chemical etchant. 